\title{
ArcheoSciences
}

Revue d'archéométrie

42-1 | 2018

Varia / 40 ans de colloques du GMPCA

\section{Dater, une histoire qui date !}

Dating, an old story!

\section{Pierre Guibert}

\section{(2) OpenEdition}

Journals

\section{Édition électronique}

URL : https://journals.openedition.org/archeosciences/5390

DOI : 10.4000/archeosciences.5390

ISSN : 2104-3728

\section{Éditeur}

Presses universitaires de Rennes

\section{Édition imprimée}

Date de publication : 27 juin 2018

Pagination : 85-101

ISBN : 978-2-7535-7587-5

ISSN : 1960-1360

\section{Référence électronique}

Pierre Guibert, «Dater, une histoire qui date ! », ArcheoSciences [En ligne], 42-1 | 2018, mis en ligne le 27 juin 2020, consulté le 01 février 2022. URL : http://journals.openedition.org/archeosciences/5390 ;

DOI : https://doi.org/10.4000/archeosciences.5390 


\title{
Dater, une histoire qui date!
}

\author{
Dating, an Old Story!
}

\author{
Pierre GuiberT ${ }^{a}$
}

\begin{abstract}
Résumé : Nous tentons dans cet article de présenter l'évolution des méthodes physiques de datation au cours des dernières décennies en mettant en lumière les facteurs qui ont été à l'origine de cette évolution. Nous en présentons les conséquences sur la pratique de la datation et sur l'évolution des problématiques de la datation en archéologie. Ainsi, nous avons passé en revue trois types de méthodes selon le mode d'enregistrement du temps : 1) variations de paramètres environnementaux : dendrochronologie et archéomagnétisme; 2) filiation radioactive : datation par les isotopes cosmogéniques, dont le radiocarbone, et datation par accumulation d'isotopes fils : potassium-40 et déséquilibre des séries de l'uranium $\left.\left({ }^{238} \mathrm{U}_{-}{ }^{234} \mathrm{U}-{ }^{230} \mathrm{Th}\right) ; 3\right)$ accumulation de charges déplacées par l'irradiation : méthodes paléodosimétriques dont la datation par luminescence et par résonance paramagnétique électronique. Selon les cas, nous évoquons des défis pour les années futures.
\end{abstract}

\begin{abstract}
My main aim in this paper is to present how the dating methods in archaeology have evolved for the last decades. We attempt at bringing into the light the factors that are at the origin of this evolution. Their consequences on the practice and the use of dating methods will be discussed, and on the changes in the chronological questions to be solved as well. Three types of methods were considered according to the way the time is recorded: 1) Variation of environmental parameters: dendrochronology, archaeomagnetism; 2) Radioactive decay: cosmogenic isotope dating including radiocarbon, and accumulation of daughters from a radioactive parent, potassium- 40 and uranium series disequilibrium dating $\left.{ }^{238} U_{-}^{234} U_{-}^{230} \mathrm{Th}\right)$; 3) Accumulation of charges displaced by irradiation: paleodosimetric techniques as luminescence and electron spin resonance dating. According to the methods, we suggest challenges for the future.
\end{abstract}

Mots clés : Archéomagnétisme, datation, dendrochronologie, déséquilibre des séries de l’uranium, isotopes cosmogéniques, luminescence, potassium-40, radiocarbone, résonance paramagnétique électronique.

Keywords: Archaeomagnetism, cosmogenic isotopes, dating methods, dendrochronology, electron spin resonance, radiocarbon, luminescence, potassium-40, uranium series disequilibrium.

\section{INTRODUCTION}

Quarante ans de datation, telle était la consigne pour le $40^{e}$ anniversaire du GMPCA. Aussi, j'ai choisi d'orienter mon propos, non pas sur quarante ans de résultats de datation, toutes périodes et aires culturelles confondues, mais plutôt sur l'évolution des méthodes de datation au cours des quatre dernières décennies, ce qui me paraissait à la fois plus intéressant et plus pédagogique pour les praticiens et les utilisateurs, et bien évidemment plus adapté au format proposé. Ce faisant, cet article ne cherche pas l'exhaustivité.
Nous ne traiterons pas de toutes les méthodes de datation, mais uniquement de méthodes physiques de datation les plus courantes ou les plus prometteuses pour l'archéologie. J'ai opéré un centrage géographique et bibliographique sur les groupes français de chronologie en archéologie, sans oublier le contexte international dans lequel nous vivons quotidiennement, car nos équipes sont petites et nous avons donc un besoin permanent de mondialisation. Nous positionnons les méthodes de datation comme un ensemble d'outils fondamentaux dans une recherche interdisciplinaire sur l'histoire de l'Homme. J'ai été aidé dans cette démarche

a CNRS, Institut de Recherche sur les Archéomatériaux - Centre de Recherche en Physique Appliquée à l'Archéologie, UMR 5060 CNRS Université Bordeaux Montaigne. Maison de l'Archéologie, Esplanade des Antilles, 33607 PESSAC cedex, France. (pierre.guibert@u-bordeaux-montaigne.fr) 
par des collègues spécialistes que j'ai interrogés et qui ont bien voulu apporter leur concours (voir les remerciements en fin d'article).

Nous laisserons d'emblée de côté les méthodes basées sur l'altération des matériaux, et en particulier sur l'hydratation des minéraux, par exemple l'hydratation des quartz ou la réhydroxylation des terres cuites qui ont fait tant couler d'encre ces dernières années (par exemple Ericson, 2004; Barrett, 2017). En fait toutes ces méthodes sont basées sur un principe d'altération différentielle, aussi permettent-elles de classer les objets selon leur degré d'altération, mais pas nécessairement selon leur âge, puisque le taux d'altération dépend fortement de facteurs environnementaux très nombreux et intrinsèques aux matériaux, mal maîtrisés, extrêmement variables dans le temps et l'espace, même à l'échelle métrique, et dont l'action n'est pas linéaire. Pour s'en convaincre, il suffit de se rappeler que l'un des paramètres sensibles pour la cinétique est la température, avec une dépendance exponentielle en inverse de la température absolue (loi d'Arrhénius) bien connue des chimistes.

S'il fallait classer les méthodes de datation, dans un souci de synthèse, il faudrait vite abandonner la dichotomie méthodes archéologiques, méthodes de laboratoire, car elles participent toutes de l'archéologie, du terrain et du laboratoire... De même, classer les méthodes selon celles qui méritent d'être détaillées car elles ont été plus largement utilisées jusqu'à présent et celles qui sont juste évoquées ne m’a pas semblé pertinent. Aussi, je présenterai plutôt les méthodes selon le processus d'enregistrement caractéristique du temps écoulé :

- Celles qui résultent de l'enregistrement de variations environnementales comme la dendrochronologie et l'archéomagnétisme;

- Celles qui résultent de la filiation radioactive, où nous distinguerons, celles basées sur les isotopes cosmogéniques produits en continu par le rayonnement cosmique (radiocarbone, béryllium-10...), et celles basées sur des isotopes de longue période présents sur Terre depuis sa formation (K-Ar ou Ar-Ar, U-Th...);

- Et enfin les méthodes paléodosimétriques, basées sur les effets cumulatifs de l'irradiation par la radioactivité naturelle : luminescence et méthodes dérivées, résonance paramagnétique électronique...

\section{LES MÉTHODES BASÉES SUR L'ENREGISTREMENT DE VARIATIONS ENVIRONNEMENTALES}

\section{La Dendrochronologie}

Chacun connaît même de manière sommaire, les principes de la méthode qui pourrait s'apparenter ainsi à un jeu d'enfant, avec le comptage des cernes de croissance. Selon l'historique de L. Tessier (2010), la première référence sur l'appréhension du rôle de l'arbre dans l'enregistrement des fluctuations climatiques est attribuée à Léonard de Vinci au $\mathrm{XV}^{\mathrm{e}}$ siècle (Stallings et al., 1937; Schulmann, 1937). La croissance de l'arbre est en effet sensible à son environnement, au climat et aux éventuels stress qu'il subit au cours de son histoire. Dans un environnement donné, chaque cerne " enregistre " par son mode de développement les conditions climatiques de l'année de sa croissance qui conditionnent ainsi son épaisseur. La succession des cernes d'un arbre du cœur vers la périphérie rend donc compte de la succession des conditions de croissance liées aux facteurs environnementaux, c'est tout au moins le signal que les dendrochronologues tentent d'isoler. Il s'agit ainsi d'un signal statistique relatif à une population d'arbres donnée et pour une espèce donnée dans un environnement global qui doit être homogène. Ainsi il s'agit d'extraire de l'analyse d'individus le signal statistique caractéristique d'une séquence climatique régionale (au sens de grande région climatique) et de remonter le temps pour construire une référence utilisable pour la dendrochronologie.

La dendrochronologie s'est développée dès la fin du XIx ${ }^{e}$ siècle en tant que discipline sous l'impulsion de deux chercheurs, Andrew E. Douglass aux États-Unis et Bruno Hüber en Allemagne qui ont posé les bases d'une pratique normalisée. C'est à partir des années 1970 que la dendrochronologie acquiert la modernité de la pratique que l'on utilise ou que l'on connaît aujourd'hui. L'étude des largeurs de cerne est fréquemment associée à des analyses complémentaires, selon les problématiques traitées, telles que l'analyse densitométrique, la répartition des isotopes légers du carbone et de l'oxygène qui forment autant d'informations liées à une chronologie de haute résolution, la plus haute que l'on puisse en effet espérer pour l'étude du passé à partir de vestiges matériels.

Depuis une quarantaine d'années, l'évolution de la dendrochronologie a suivi l'évolution technique en termes d'informatique, de numérisation et, plus récemment, de mise en réseau des données à l'échelle internationale. Ce n'est pas tant dans les concepts que la discipline a évolué mais beaucoup plus dans ses liens avec d'autres composantes scientifiques. La dendrochronologie est donc très rarement utilisée 
seule, et de nombreuses disciplines utilisent cet outil pour la reconstruction des climats, les pratiques forestières, l'étude d'impact sur la croissance des arbres de phénomènes de stress environnementaux (glissements de terrain, avalanches...) ou parasitaires, sans oublier l'archéologie, notre principal sujet de préoccupation au GMPCA. Le lecteur intéressé pourra aisément consulter l'ouvrage collectif dirigé par L. Astrade et C. Miramont (2010) pour un aperçu des applications de la dendrochronologie dans de très nombreux domaines.

Les capacités de la dendrochronologie en termes de résolution temporelle ont été très rapidement perçues et exploitées dans la communauté de la préhistoire "récente " (Lambert et Lavier, 1990). Elles sont ainsi clairement démontrées avec la chronologie d'habitats lacustres néolithiques dont l'étude détaillée a permis, par exemple, d'accéder aux modalités d'exploitation des terres et de gestion des ressources par les néolithiques des lacs de Clairvaux et Chalain dans le Jura (Pétrequin 2005). De nombreuses autres applications associées à l'archéologie des milieux humides et littoraux, études des aménagements portuaires, des aménagements de cours d'eau peuvent être citées (Billamboz, 2014; Bernard et Langouet, 2014) de même que dans le domaine de l'archéologie navale (Guibal, 1992). En archéologie du bâti, l'étude des charpentes et des bois d'œuvre offre un large spectre de recherches pour l'histoire des techniques constructives et de maintenance des édifices (Hoffsummer, 1999, 2002; Maggi et al., 2002).

Les défis actuels pour la dendrochronologie visent à l'extension de la séquence chronologique. La préoccupation des spécialistes concerne les hétéro-connexions, c'est-à-dire les corrélations interspécifiques en particulier la corrélation chêne/pin qui permet une séquence chronologique jusque vers 12600 ans avant le présent (Friedrich et al., 2004; Miramont et al., 2010), ce qui couvre l'histoire humaine depuis la fin du Paléolithique Supérieur. Les espoirs de continuer la séquence sont fondés sur le recouvrement de chronologies alpines flottantes jusque vers 16000 avant le présent. Il serait ainsi possible de remonter jusqu'au Magdalénien Moyen en Europe occidentale. Pour le moment des problèmes de synchronisation des séquences subsistent, cependant la possibilité demeure de mettre au jour des bois suffisamment bien conservés pour atteindre ces objectifs. L'idée est en effet de poursuivre la calibration de haute résolution du radiocarbone indépendamment des mesures couplées avec le déséquilibre des séries de l'uranium d'échantillons marins dont l'effet réservoir n'est pas forcément constant dans le temps et l'espace.

\section{L'archéomagnétisme}

Je ne ferai qu'évoquer ce que les chronologues nomment paléomagnétisme, à savoir le repérage de la succession des renversements de polarité du champ magnétique terrestre comme marqueurs chronologiques dans les stratigraphies quaternaires. Ces différents événements de renversements forment des jalons pour le Pliocène et le Pléistocène qui sont largement utilisés en Paléontologie (Sémah et al., 2000).

C'est Emile Thellier après sa thèse en 1938 (Thellier, 1938) et son épouse Odette qui posent les bases du magnétisme des terres cuites et leur application à la chronologie (Thellier et Thellier, 1959). Deux articles de synthèse de 1966 (Thellier, 1966; 1981) dressent le bilan de l'archéomagnétisme en France et tentent de dégager les grandes lignes de recherche pour le futur, et ce que l'on peut dire aujourd'hui c'est qu'elles ont été suivies et restent toujours d'actualité. Sur le principe, la méthode de datation repose sur la connaissance de l'évolution du champ magnétique terrestre et son enregistrement dans les roches chauffées et les terres cuites par aimantation des minéraux magnétiques. Au refroidissement des matériaux, l'aimantation induite se fige durablement et le matériau présente donc une aimantation dite thermorémanente qui est parallèle au champ géomagnétique ambiant. Au cours du temps, le champ magnétique terrestre (CMT) évolue en direction et en intensité : on parle de variation séculaire. La datation par archéomagnétisme repose ainsi, dans la majorité des cas jusqu’à présent, sur la comparaison des caractéristiques directionnelles de l'aimantation thermorémanente d'un échantillon à une courbe de calibration, c'est-à-dire l'inclinaison (angle entre le plan horizontal et la direction du CMT) et la déclinaison (angle entre le Nord magnétique et le Nord géographique dans un plan horizontal) (Gallet et al., 2009; Hervé et Lanos, 2013). Nous ajouterons que depuis quelques années l'intensité est le $3^{\mathrm{e}}$ élément du vecteur qui est venu s'ajouter aux données angulaires. La courbe de calibration est construite pour les périodes précédant les observations et mesures systématiques directes du CMT dans les observatoires, à partir d'échantillons archéologiques bien datés et en position primaire, c'est-à-dire non déplacés par rapport à leur position de chauffe (éléments de four, aires de combustion, etc.). Les courbes de référence sont valables pour une région géographique donnée, car le CMT présente des variations locales non dipolaires. La notion de région représente la zone géographique pour laquelle il est possible d'apporter des corrections standard en direction et intensité grâce à un modèle dipolaire local. Cela représente par exemple en France, plusieurs centaines de kilomètres autour de Paris, site généralement utilisé comme référence centrale, cependant largement 
excentré géographiquement par rapport au territoire métropolitain (je propose de choisir Garchy dans la Nièvre avec plus de pertinence géographique et historique, car cela a été le siège du GMPCA depuis ses débuts jusque dans les années 2000 !). La datation par archéomagnétisme doit donc répondre à deux problématiques : premièrement, la mesure fiable de l'orientation du champ ancien et de son intensité, deuxièmement, et non la moindre des problématiques, la construction de courbes de calibration qui nécessite que les datations des objets de référence soient particulièrement fiables et précises.

Il y a 40 ans, les bases de l'archéomagnétisme étaient déjà posées. À Rennes, la mise en place d'un premier laboratoire d'archéomagnétisme à vocation purement archéologique a été réalisée dans les années 80 par notre regretté collègue Loïc Langouet et ses collaborateurs. À cette époque, en France, la dynamique patrimoniale était objectivée par la constitution de la carte archéologique du territoire sous l'impulsion des découvertes issues de la prospection au sol et aérienne de l'habitat rural gallo-romain. Aussi le groupe de Rennes a-t-il mis en place une méthode de datation des matériaux déplacés (de leur lieu de production), en particulier, les terres cuites architecturales (TCA : briques, tegulae...) grâce au fait que leur positionnement dans un four gallo-romain résultait d'un empilement régulier sur une sole horizontale. L'analyse de l'orientation de l'aimantation thermorémanente de ces objets rend possible la détermination de leur position de cuisson (de chant, debout ou à plat) en raison des limites angulaires du cône de variation de la direction du CMT, tout au moins sous nos latitudes. En étudiant la répartition de l'orientation du champ sur une centaine d'échantillons d'un même lot ou d'une même production, il est possible ainsi de déterminer avec précision l'inclinaison du CMT au moment de la manufacture de ces terres cuites (Lanos, 1987, 1990, 1994). Si l'on fait un bilan sur les avancées en matière de mesure du champ magnétique thermorémanent, nous devons citer également les affinements importants de la méthode avec la prise en compte et la correction des effets d'anisotropie magnétique (directions d'aimantation préférentielles de certains minéraux) sur les directions de l'aimantation thermorémanente qui peut alors ne pas être parallèle au champ géomagnétique (Veitch et al., 1984; Lanos, 1987, 1999; Goulpeau et al., 1989).

La construction des courbes de référence locales, de portée régionale, est une préoccupation permanente pour les géomagnéticiens (Lanos, 2004; Gomez-Paccard et al., 2012, 2016; Gallet et al., 2009; Genevey et al., 2013, 2016; Hervé et al., 2013a, 2013b, 2017 pour les compilations les plus récentes en Europe occidentale). L'objectif est de caractériser les fluctuations du CMT et ses temporalités. La collaboration avec les archéologues pour « fournir » des sites bien datés est absolument nécessaire. En retour l'archéologue et le chronologue doivent porter un regard critique sur l'établissement de la chronologie, ce qui n'a rien d'évident, car il faut remonter aux sources de la chaîne d'inférences ayant abouti aux attributions chronologiques. En France et en général en Europe romaine, la période antique est très bien documentée, de même que le "bas " Moyen Âge et la période moderne. Cependant le "désert " du haut Moyen Âge (peu de constructions reconnues comme telles de cette époque) a tardé à être "peuplé », et il a fallu attendre la première décennie 2000 pour que des études plus systématiques de TCA issues de bâtiments soient entreprises, en France en partie sous l'impulsion du groupe de recherche européen CNRS sur les Terres Cuites Architecturales, avec un couplage systématique interméthodes et archéologie du bâti sur une vingtaine de sites dont la construction est comprise entre le $\mathrm{v}^{\mathrm{e}}$ et le $\mathrm{XI}^{\mathrm{e}}$ siècle. La publication de ces données par I'IRAMAT, Géosciences Rennes et l'Institut de Physique du Globe de Paris est une priorité pour les saisons à venir.

La mesure de l'intensité du champ magnétique comme

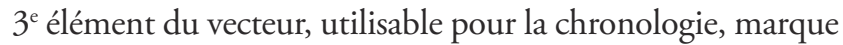
un effort important de la communauté au cours de la dernière décennie pour documenter les variations d'intensité du CMT depuis l'âge du Bronze moyen (figure 1, Hervé et al., 2013b, 2017). Des difficultés ont cependant été rencontrées avec la détermination du CMT ancien à partir de l'aimantation thermorémanente. La mesure de l'intensité suppose une désaimantation thermique suivie d'une réaimantation. La régénération du signal magnétique est altérée par les conditions RedOx de la chauffe en laboratoire qui divergent le plus souvent des conditions archéologiques, les minéraux magnétiques étant particulièrement sensibles à la composition de l'atmosphère au cours des traitements thermiques (pression partielle d'oxygène, de $\mathrm{CO}_{2}$, de $\mathrm{CO}$, de vapeur d'eau $\mathrm{H}_{2} \mathrm{O}$ etc.). On retrouve dans les phénomènes de magnétisation, une sensibilité aux conditions de chauffe analogue à celle observée en thermoluminescence qui justifie des protocoles de mesures complexes. L'intensité d'aimantation thermorémanente est par ailleurs dépendante de la vitesse de refroidissement, ce qui entraîne une source de variabilité supplémentaire dans les mesures effectuées en laboratoire pour se rapprocher des conditions de refroidissement compatibles avec celles des objets étudiés.

Parmi les recherches exploratoires en cours en archéomagnétisme, l'application du magnétisme à des objets non chauffés tels que des briques d'adobe, ou à des pigments à base d'oxydes de fer suscite un vif intérêt en raison de son potentiel pour l'archéologie. On suppose qu'une orientation préférentielle des minéraux magnétiques s'opère 
Figure 1 : Variation de l'intensité du champ magnétique terrestre au cours des 3500 années passées (inédit, calcul de courbe par Ph. Lanos et compilation des données par G. Hervé - données existantes en septembre 2017).

Figure 1: Variation of the geomagnetic field intensity for the last 3,500 years (unpublished data: compilation of existing data by $G$. Hervé and curve calculation by $\mathrm{Ph}$. Lanos, september 2017).

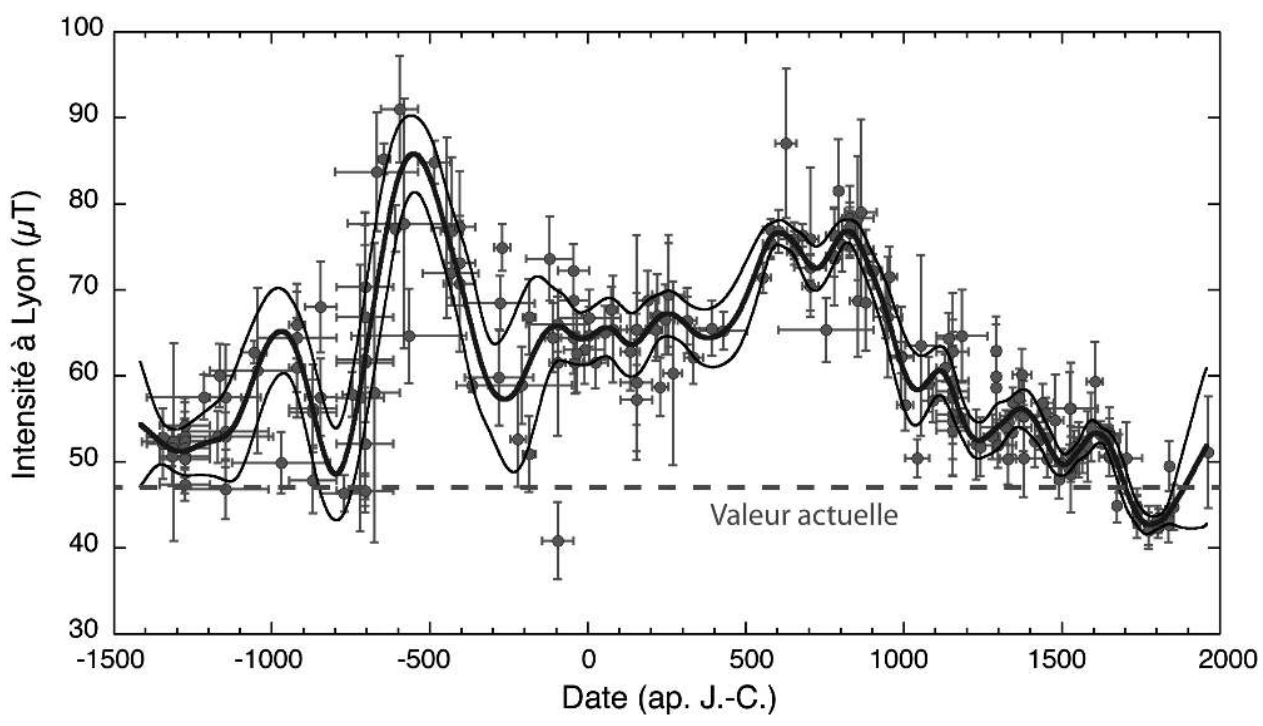

lorsque ceux-ci sont en suspension en phase aqueuse et que le séchage fige les configurations. Cependant on connaît mal les effets de la rugosité du support et d'autres irrégularités de surface sur l'orientation finale des grains.

La poursuite de la construction des courbes d'étalonnage reste une priorité pour les archéo- et géomagnéticiens, à partir d'objets "bien " datés par des méthodes variées et convergentes afin de compléter les séries temporelles sur différentes aires géographiques. Une vaste base de données internationale sur les sites et structures de référence servant à établir la courbe d'étalonnage est en cours, intégrant une évaluation critique des datations dites de référence.

\section{Méthodes Basées \\ SUR LA FILIATION RADIOACTIVE}

Un grand nombre de méthodes de datation sont basées sur la filiation radioactive. Parmi celles-ci nous considèrerons quelques exemples de méthodes liées à l'exposition de la surface du globe au rayonnement cosmique et dépendant ainsi d'une production continue d'isotopes cosmogéniques, comme le radiocarbone, le béryllium-10, et le chlore-36, et quelques exemples de méthodes basées sur des couples géochimiques en filiation radioactive dont au moins le parent est introduit dans la roche ou dans le matériau au moment de sa formation, comme les méthodes au potassium- 40 et celles basées sur le retour à l'équilibre séculaire des séries de l'uranium.

\section{Radiocarbone}

La datation par radiocarbone est assurément la méthode la plus populaire en archéologie et celle qui a suscité le plus d'efforts au niveau mondial depuis les années 1950 (Libby et al., 1949) et le prix Nobel de Chimie de Libby en 1960. Chacun connaît le principe de la méthode que nous nous contenterons d'évoquer ici. La formation de carbone-14 à partir des réactions nucléaires entre le rayonnement cosmique et les molécules d'azote en est à l'origine. Les atomes ainsi formés sont immédiatement engagés dans des molécules de $\mathrm{CO}_{2}$ par interaction chimique avec l'ozone. Les mouvements atmosphériques assurent le brassage à l'échelle terrestre des molécules ainsi naturellement marquées. Les organismes vivants étant en équilibre avec l'atmosphère, le rapport ${ }^{14} \mathrm{C} /{ }^{12} \mathrm{C}$ est conservé entre la source, l'atmosphère, et le récepteur, tant que l'organisme est vivant. Cet équilibre cesse à la mort de l'organisme, et la décroissance radioactive du radiocarbone dans les restes de l'organisme démarre. J'attire cependant l'attention du lecteur que cette façon d'exprimer les choses peut être à l'origine d'erreurs d'interprétation parmi nos étudiants. En fait tout dépend de ce que nous considérons sous le terme organisme : il s'agit bien évidemment de parties d'un être vivant qui disposent des capacités de renouvellement de ses molécules jusqu'à la mort de l'individu. Ainsi, cette définition ne peut s'appliquer complètement aux végétaux et c'est là la source d'erreur. Le charbon de bois ne date pas la mort de l'arbre, mais plus exactement il date la formation de la cellulose, longues chaînes carbonées qui assurent la structure du bois et qui ne se renouvellent plus une fois formées. Ainsi dater un charbon de bois revient à dater la formation des cellules 
végétales correspondantes qui seront ensuite soumises à la mesure radiocarbone par les archéologues ou par d'autres spécialistes. La preuve ? Il serait impossible de faire des corrections dendrochronologiques si tel n'était pas le cas et nous entendrions moins parler d'effet vieux bois lorsque la datation radiocarbone est plus ancienne qu'attendue!

Les 40 dernières années ont été jalonnées par plusieurs faits marquants en termes d'évolution de l'instrumentation, de progrès dans la justesse, et dans la diversité de nature des échantillons datables. En premier lieu, ce sont manifestement les progrès technologiques qui ont permis au radiocarbone le développement que nous avons connu ces dernières décennies, grâce, entre autres, à la mise au point d'accélérateurs tandem à la fin des années 1970, après l'article considéré comme fondateur de R.A. Muller (1977) pour le comptage direct des atomes et non des désintégrations radioactives béta. En France, l'installation du Tandétron à Gif-sur-Yvette en 1981, puis la mise en place de la plateforme ARTEMIS en 2003 ont profondément marqué le paysage archéologique ainsi que les pratiques de la datation dans notre communauté. La spectrométrie de masse par accélérateur (SMA) a rendu possible l'analyse de très petites quantités de carbone, quelques milligrammes, ce qui a permis, parmi les exemples les plus connus, d'accéder directement à la datation du carbone végétal utilisé comme pigments dans les représentations de grottes ornées, notamment à Chauvet Pont d'Arc en Ardèche (Valladas et al., 2005). Aujourd'hui encore, les deux systèmes de mesure du radiocarbone, le comptage béta classique des désintégrations béta et le SMA coexistent. Plus récemment, la miniaturisation des spectromètres de masse permet maintenant de proposer des équipements de faible encombrement. Ce sont des appareillages de type MICADAS (acronyme pour MIni CArbon DAting System) (Synal et al., 2007; Bard et al., 2015) utilisables dans des locaux de quelques dizaines de $\mathrm{m}^{2}$.

Comme chacun sait, l'hypothèse de Libby concernant la constance de la production du radiocarbone par le rayonnement cosmique s'est avérée fausse dès que la précision des mesures est devenue suffisante, dès la fin des années 1950. Cela provient des variations temporelles de l'intensité du rayonnement cosmique et de ses interactions dans la haute atmosphère. Des corrections ont donc été apportées aux datations avec l'analyse couplée par dendrochronologie et radiocarbone sur les fameuses cellules végétales issues des cernes de croissance d'arbres bien datés (Friedrich et al., 2004). Lors de la création du GMPCA, en 1977, la calibration dendrochronologique permettait de corriger jusque vers 10000 ans avant le présent. Grâce à l'effort international, l'extension des possibilités de comparaison à des formations coralliennes, ou à des formations stalagmi- tiques par couplage U/Th et radiocarbone et le comptage de varves organiques permettent aujourd'hui de proposer des corrections sur toute la portée du radiocarbone, soit sur les 50000 dernières années (Reimer et al., 2013). Cependant il faut bien noter que par rapport à une correction dendrochronologique, la précision de la calibration est moindre, et des incertitudes subsistent encore sur l'importance des effets-réservoirs de certaines séries de formations carbonatées prises en compte dans l'expérience IntCal2013. C'est la raison pour laquelle l'extension des corrections dendrochronologiques, notamment avec les séries chronologiques alpines encore flottantes conserve tout son intérêt pour la calibration du radiocarbone.

En termes de nature d'échantillons datables, par rapport à la fin des années 1970, de nouvelles possibilités existent liées à la réduction considérable du volume nécessaire avec l'usage de la spectrométrie de masse par accélérateur. Ainsi, comme nous l'avons déjà signalé, la possibilité de dater directement les pigments a véritablement mobilisé la communauté des pariétalistes, particulièrement lorsque les premières dates de Chauvet et de Cosquer ont été obtenues. Les controverses qui sont apparues (on trouvera la synthèse assez détaillée publiée par Pettitt, 2008) ont induit des efforts méthodologiques. En plus d'expériences d'intercomparaison spécifiques mises en œuvre à Chauvet (les intercomparaisons sont pratiquées régulièrement depuis des décennies par les spécialistes du radiocarbone), d'autres méthodes en complémentarité du radiocarbone, comme le déséquilibre des séries de l'uranium, le Chlore-36 et même la luminescence ont été mobilisées sur ce site (Quilès et al., 2014; Genty et al., 2004; Sadier et al., 2012; Guibert et al., 2015).

C'est aussi cette réduction des quantités de matière qui permet aujourd'hui de dater les aciers médiévaux, dans les zones du métal les plus fortement carburées (Leroy et al., 2015) ce qui ouvre ainsi de nouvelles possibilités d'étude pour l'histoire des monuments lorsqu'il s'agit de renforts ou d'éléments métalliques pour l'architecture, et plus généralement pour l'économie de la production métallique jusqu'à ce que la houille fasse son apparition. La datation de la carbonatation des mortiers, évoquée comme possible dans les années 1960 (Labeyrie et Delibrias, 1964), résiste quelque peu encore à un développement systématique dans l'archéologie du bâti (Hajdas et al., 2017). Malgré la mise en œuvre de protocoles d'attaque chimique séquentielle des carbonates, les incertitudes demeurent. Un effort particulier de caractérisation des formations carbonatées au sein des mortiers est en cours dans le microcosme des spécialistes qui fondent leurs espoirs notamment sur l'analyse des aragonites d'origine pyrogénique (Xu et al., 2016; Toffolo et al., 2017). 


\section{Les isotopes du béryllium, de l'aluminium et du chlore et l'archéologie}

Avec des demi-vies respectives de 1,36Ma, 730ka, 300ka, les isotopes ${ }^{10} \mathrm{Be},{ }^{26} \mathrm{Al}$, et ${ }^{36} \mathrm{Cl}$, permettent de remonter plus loin dans le temps que les méthodes que nous avons évoquées jusqu'à présent et ils permettent également de dater d'autres types d'événements. Ainsi le béryllium-10, comme le radiocarbone, est produit dans la haute atmosphère par interaction de neutrons avec les noyaux d'azote. Il est aussi produit dans les roches de surface par interaction de muons avec l'oxygène. Les nucléides ${ }^{26} \mathrm{Al}$ et ${ }^{36} \mathrm{Cl}$ sont produits quant à eux dans les roches de surface à partir respectivement du silicium-28, et du calcium-40. Ainsi deux modes de production distincts, atmosphérique et terrestre ("in situ »), impliquent deux ensembles possibles de processus datables.

Le béryllium-10 atmosphérique est fixé par les aérosols puis arrive à la surface de la terre emporté dans les précipitations atmosphériques. Une partie du béryllium s’adsorbe à la surface de grains dans des sédiments lacustres ou marins et se retrouve donc dans la phase dite authigène des sédiments. Parallèlement le béryllium-9 (noyau stable) est libéré par l'érosion des roches, une partie se dissout puis s'adsorbe également en surface des grains (Bourlès et al., 1989). Selon notre collègue $\mathrm{D}$. Bourlès du CEREGE, dont je reprends ici les propos, il est bien évidemment nécessaire de normaliser la concentration mesurée en ${ }^{10} \mathrm{Be}$ par celle en ${ }^{9} \mathrm{Be}$ car divers processus physico-chimiques altèrent quantitativement l'information sur la production initiale de ${ }^{10} \mathrm{Be}$. «Étant donné les voies d'introduction très différentes $\mathrm{du}{ }^{10} \mathrm{Be}$ et $\mathrm{du}$ ${ }^{9} \mathrm{Be}$ dans l'environnement terrestre, il apparaît que seule la forme soluble de ces deux isotopes peut être homogénéisée avant adsorption sur les particules et dépôt des sédiments. Adsorbés à la surface des particules dans un rapport représentatif de la masse d'eau qui les contenait, les isotopes ${ }^{9} \mathrm{Be}$ et ${ }^{10} \mathrm{Be}$ participent dès lors à la constitution de la phase authigène des sédiments, c'est-à-dire la phase formée à partir des éléments dissous. Seule cette phase authigène des sédiments est considérée pour la datation ». Pour en savoir davantage le lecteur intéressé par les aspects méthodologiques de la datation par le béryllium-10 pourra ainsi se référer à l'article détaillé d'Anne Elisabeth Lebatard et al. (2009) qui présente la démarche qui a abouti à la datation à environ 7 millions d'années d'Hominidés Mio-pliocènes du Tchad. L'une des problématiques de la démarche est de s'assurer que dans un bassin versant donné, le rapport ${ }^{10} \mathrm{Be} /{ }^{9} \mathrm{Be}$ est constant.

La production " au sol » d'isotopes cosmogéniques donne également lieu à de nombreuses applications : âge d'exposition de roches au rayonnement cosmique, par exemple pour la détermination des vitesses de dénudation, le retrait de glaciers (Brown et al., 1991), l'effondrement de falaises etc. ou bien à l'inverse il est possible de déterminer l'histoire de roches anciennement exposées puis de nouveau enfouies (Shen et al., 2012). Des situations archéologiques variées peuvent bénéficier d'approches chronologiques de ce type. L'exemple de la chronologie des occupations humaines à la Grotte Chauvet Pont-d'Arc est emblématique du concours de la datation par le chlore-36. Il a ainsi été montré que l'entrée paléolithique de la grotte avait été obturée à la suite de plusieurs épisodes d'effondrement de la falaise qui la surplombait, notamment, le dernier vers $22 \mathrm{ka}$ avant le présent qui scelle définitivement le réseau karstique et ses représentations pariétales (Sadier et al., 2012). Les lecteurs intéressés par la datation de l'occupation humaine à Chauvet Pont d'Arc pourront aussi lire la synthèse des datations dans l'article d'Anita Quilès et al. (2016). Enfin, pour des périodes beaucoup plus proches de nous, on peut proposer comme défi pour la datation au chlore-36 la datation des carrières anciennes (âge d'exposition au rayonnement cosmique des fronts de taille dans des calcaires), ce qui contribuerait à caractériser plus sûrement les sources de matériau de construction pour l'Antiquité et le Moyen Âge (Gutiérrez Garcia-Moreno, 2014).

\section{Datation par la filiation ${ }^{40} \mathrm{~K}-{ }^{40} \mathrm{Ar}$}

La méthode de datation basée sur la filiation du potassium 40 est utilisée en géochronologie pour dater la formation de roches contenant du potassium, c'est une évidence, et capables de retenir l'Argon-40 formé. L'une des premières mentions de la possibilité d'utiliser cette filiation en géochronologie a été publiée en 1948 par les physiciens Aldrich et Nier de l'université du Minnesota (Gillot et al., 1982; Aldrich et Nier, 1948). Aujourd'hui c'est une méthode de choix pour la chronologie du volcanisme (Lefevre et Gillot, 1994; McDougall, 2014). La méthode est basée sur la mesure du rapport ${ }^{40} \mathrm{~K} /{ }^{40} \mathrm{Ar}$ dans le minéral étudié, rapport qui évolue et décroît au cours du temps. Deux techniques sont employées : le Potassium-Argon et l'Argon-Argon.

Dans le cas du Potassium-Argon, la mesure de l'argon- 40 produit au cours du temps se fait sur un aliquote de l'échantillon par spectrométrie de masse et la mesure du potassium sur un second aliquote par spectrométrie d'émission atomique après mise en solution des minéraux.

La technique dite Argon-Argon est basée sur la détermination au cours de la même mesure du rapport ${ }^{39} \mathrm{Ar} /{ }^{40} \mathrm{Ar}$ par spectrométrie de masse, ${ }^{39} \mathrm{Ar}$ étant produit par activation neutronique de ${ }^{39} \mathrm{~K}$ par des neutrons rapides. Pour une irradiation neutronique donnée, la quantité de ${ }^{39} \mathrm{Ar}$ produite est donc directement proportionnelle à la concentra- 
tion en ${ }^{39} \mathrm{~K}$ dans le minéral, isotope lui-même en rapport isotopique constant avec ${ }^{40} \mathrm{~K}$ (on notera que l'abondance isotopique de ${ }^{40} \mathrm{~K}$ varie nécessairement avec le temps en raison de la décroissance radioactive de ${ }^{40} \mathrm{~K}$ initial de l'écorce terrestre). On détermine donc avec le même équipement de spectrométrie de masse et au cours de la même série expérimentale le rapport ${ }^{39} \mathrm{Ar} /{ }^{40} \mathrm{Ar}$ dans la roche échantillon et dans une roche étalon (datée elle-même par la technique Potassium-Argon). Un chauffage par palier de température des minéraux est effectué jusqu'à fusion totale pour dégazer et mesurer l'argon piégé. Cette procédure permet de, entre autres, contrôler la validité du chronomètre : perte d'argon par diffusion thermique (néotectonique) ou perte de potassium par altération chimique du minéral (lixiviation). La demi-vie de ${ }^{40} \mathrm{~K}$ étant de $(1,251 \pm 0,003) 10^{9}$ ans ${ }^{1}$, la méthode a longtemps été appliquée au volcanisme ancien. Son utilisation pour l'archéologie est relativement récente et elle est liée à l'amélioration des spectromètres de masse dans les années 1990 et à l'affinement des protocoles de préparation et d'étude des échantillons (Deino et al., 1998). Il est ainsi possible de dater indirectement par cette méthode des dépôts sédimentaires contenant des minéraux volcaniques. En fait, on date la formation des minéraux volcaniques associés au sédiment quand on peut effectivement assurer que ces minéraux éruptifs sont issus d'événements géographiquement proches (à portée de panaches) contemporains (dépôts de cendres notamment). L'équipe de V. Michel a ainsi proposé une réévaluation de la chronologie du site d'Orgnac 3, en Ardèche, emblématique d'industries lithiques du début du Paléolithique Moyen. La datation Ar-Ar sur sanidine a contribué à ce renouvellement en fournissant un ensemble de datations cohérentes et précises autour de $300 \mathrm{ka}$ pour le niveau 2 dans la partie supérieure de la séquence. Elle a conduit les auteurs à considérer que ce site renferme les manifestations parmi les plus précoces de la technique de débitage Levallois et donc du Paléolithique Moyen (Michel et al., 2013).

La datation d'événements volcaniques récents par la méthode Ar-Ar est possible et peut évidemment intéresser l'archéologie des périodes Holocène, voire des périodes historiques. Nous citerons les tentatives de "dater " l'éruption de 79AD du Vésuve (Renne et al., 1997) qui a amené les auteurs aux limites de la méthode, ici basée sur l'analyse de sanidines de dimensions millimétriques extraits de ponces volcaniques. D'autres auteurs ont par la suite utilisé les émissions minérales de l'éruption plinienne du Vésuve pour de nouveau tester la méthode, avec un regard critique

1. selon les publications les plus récentes [https://www.nndc.bnl.gov/ chart consulté 16.11.2017]. sur les résultats de leurs prédécesseurs. C’est ce que proposent Lanphere et ses collaborateurs dans une publication de 2007, en obtenant des résultats tout à fait concordants avec les données historiques, ce qui leur permet de statuer sur la validité de la méthode pour des anciennetés de l'ordre de 2000 ans.

Techniquement, grâce à la sensibilité accrue de nouveaux équipements de spectrométrie de masse, il devient possible d'examiner des grains individuels dont les dimensions sont de l'ordre de $300 \mu \mathrm{m}$. On accède ainsi, comme en datation par luminescence, à une extension des possibilités de datation à des milieux où l'on soupçonne la possibilité de mélange de grains d'origine différente et donc d'âges différents.

\section{Datation par le déséquilibre des séries de l'uranium}

Il existe de nombreuses variantes de la datation par le déséquilibre des séries de l'uranium selon les plages de temps considérées, et donc selon les isotopes concernés et également selon le mode de mesure de l'activité des couples parents-fils. Nous nous intéresserons ici à la datation par le retour à l'équilibre séculaire des isotopes ${ }^{234} \mathrm{U}$ (période radioactive de $245 \mathrm{ka}$ environ) et ${ }^{230} \mathrm{Th}$ (période de $75 \mathrm{ka}$ ) et à leur utilisation pour la datation des formations carbonatées. On notera que cette méthode, que nous convenons d'appeler U-Th, est aussi utilisée pour dater des ossements fossiles. Nous reviendrons sur les questions particulières qui se posent pour ces biomatériaux (Millard et Hedges, 1996).

En archéologie, l'usage le plus fréquent de la méthode concerne la datation de spéléothèmes. Le principe repose sur les propriétés de solubilité différentielle entre l'uranium et ses radioéléments-fils. Ainsi, pour résumer une chimie plutôt complexe et plutôt bien connue (Rosholt et al., 1966; Rosholt, 1982; Gascoyne, 1982), nous considérons que l'uranium dans son état d'oxydation le plus élevé (+VI) forme des ions très solubles, alors que les ions du thorium (état $+\mathrm{IV}$ ) sont quasiment insolubles dans les conditions naturelles de $\mathrm{pH}$. Le lessivage des sols par les eaux de ruissellement et d'infiltration altère les minéraux qui, à leur tour, libèrent des éléments dont les plus solubles sont entraînés dans la phase aqueuse. En milieu karstique, l'eau d'infiltration se charge d'ions calcium et d'ions carbonates. Sous certaines conditions de $\mathrm{pH}$ et de teneur en gaz carbonique, dans les cavités, les carbonates de calcium précipitent piégeant des ions d'uranium présents dans l'eau. Le carbonate de calcium formé contient donc initialement à l'état de trace des ions d'uranium $\left({ }^{238} \mathrm{U},{ }^{235} \mathrm{U},{ }^{234} \mathrm{U}\right)$, mais pas de thorium. Au fil du temps, le ${ }^{230} \mathrm{Th}$ produit par ${ }^{234} \mathrm{U}$ s'accumule et parallèlement les désintégrations de ${ }^{234} \mathrm{U}$ sont compensées 
par celles de ${ }^{238} \mathrm{U}$, isotope parent à plus longue période. Le rapport ${ }^{230} \mathrm{Th} /{ }^{234} \mathrm{U}$ passe de 0 à l'origine des temps et tend asymptotiquement vers 1 . Une équation d'âge reliant ${ }^{238} \mathrm{U}$, ${ }^{234} \mathrm{U}$ et ${ }^{230} \mathrm{Th}$ permet de déterminer l'âge de la formation carbonatée (Couchoud et al., 2009; Ghaleb et Falguères, 2017). Cette méthode de datation suppose que le matériau ne s'altère pas au cours du temps et ne soit pas sujet à des incorporations ou déplétions en uranium après sa formation, comme cela est fréquemment le cas avec les ossements. On parle de milieu fermé et l'une des problématiques de la méthode concerne l'intégrité du matériau. Aussi c'est la raison pour laquelle la qualification d'un résultat de datation passe nécessairement par une caractérisation fine des objets datés (observations microscopiques, étude de composition, de structure et de texture, etc.).

En 1977, la technique de mesure utilisée pour la datation U-Th était quasi exclusivement la spectrométrie alpha. Lorsque les spectromètres de masse couplés à la thermoionisation des cibles échantillons ont pu être mis en œuvre dans les années 1980, la qualité des mesures a fait un bond quantitatif, la précision et la sensibilité ayant été accrues d'au moins un ordre de grandeur (d'une incertitude de l'ordre de $5 \%$ voire davantage en spectrométrie alpha, on atteint moins de $1 \%$ par TIMS : thermo-ionisation mass spectrometry), ce qui ouvrait aussi la voie à des applications de plus en plus fines de la méthode et permettait d'établir des chronologies avec une bien meilleure résolution chronologique. Pour donner une idée de ce gain, nous nous sommes intéressés à la datation de planchers stalagmitiques à la Chaise de Vouthon en Charente, dans l'abri Bourgeois Delaunay. Deux planchers stalagmitiques, le plancher 7 pour le niveau supérieur, le plancher 11 pour le niveau inférieur, encadrent des niveaux anthropiques moustériens dont on cherche à connaitre l'intervalle chronologique. B. Blackwell, H. Schwarcz et A. Debénath en 1983 publient les datations U-Th par spectrométrie alpha de ces formations : les datations du plancher 11 varient de $150 \pm 15$ à $112 \pm 7 \mathrm{ka}$, celles du plancher 7 supérieur varient de $114 \pm 7$ à $71 \pm 6$. Les données sont reprises par I. Couchoud au cours de son doctorat dans les années 2000 et elle effectue à l'issue d'une caractérisation fine de ses échantillons des micro-prélèvements dans les lamines des mêmes planchers qui sont datées par la technique TIMS (Couchoud et al., 2009). Les résultats obtenus pour le plancher 11 convergent vers un démarrage de croissance vers $127,1 \pm 1,5 \mathrm{ka}$ puis une fin de croissance à $113 \pm 1 \mathrm{ka}$, en revanche elle montre que le plancher 7 s'est formé assez rapidement il y a $78 \pm 1$ ka. Les occupations humaines sont donc encadrées par les limites de l'intervalle $113 \pm 1$ à $78 \pm 1 \mathrm{ka}$. Outre la définition précise du cadre chronologique de la présence anthropique à l'abri
Bourgeois-Delaunay, c'est l'accès à des informations chronologiques de haute résolution qui est rendu possible, ce que montrent les datations obtenues sur les lamines du plancher 11 qui sont cohérentes avec l'ordre de croissance déterminé par l'observation microscopique. Les paléoclimatologues ne s'y sont pas trompés et utilisent les spéléothèmes comme témoins fiables pour l'étude des paléoclimats continentaux (par ex. Quinif et al., 1994; Couchoud et al., 2009). Nous noterons qu’à partir des années 1990, la précision atteinte par les mesures par TIMS ont contribué très significativement à la calibration du radiocarbone au-delà des séries dendrochronologiques grâce à l'étude couplée ${ }^{14} \mathrm{C} / \mathrm{U}-\mathrm{Th}$ de formations coralliennes (Bard et al., 2013; Reimer et al., 2013). Il faut noter cependant que l'une des incertitudes qui ont été rencontrées dans cette intercalibration concerne la variabilité de l'effet réservoir en carbone à partir desquels les coraux se nourrissent pour se développer.

Les ouvertures actuelles de la méthode sont liées à l'amélioration de la sensibilité des techniques de spectrométrie de masse et de prise de matière sur l'échantillon. Au-delà du fait que cette méthode est parfaitement utilisable pour des périodes récentes (formations stalagmitiques observées sur des ouvrages bâtis gallo-romains, notamment des aqueducs, pour dater leur utilisation ultime au Moyen Âge : PonsBranchu et al., 2014), la sensibilité avec les nouveaux systèmes d'ablation laser femto-seconde est telle qu'il devient possible de travailler sur des objets qui jusqu’à un passé relativement récent résistaient à une analyse précise. On peut citer les voiles de calcite qui recouvrent certaines représentations pariétales paléolithiques (on obtient un Terminus Ante Quem pour l'art pariétal), et la datation d'œufs d'autruche gravés dans des sites du Middle Stone Age africain (Peycheran et al., 2016, 2017). La possibilité de cartographier les isotopes ${ }^{238} \mathrm{U},{ }^{234} \mathrm{U}$ et ${ }^{230} \mathrm{Th}$ ouvre aussi de nouvelles perspectives de caractérisation de la répartition de l'uranium et de ses descendants et de datation des biomatériaux dans les zones les moins perturbées par l'adsorption-diffusion d'uranium post-dépositionnelle (pour reprendre les termes de nos collègues Millard et Hedges, 1996).

\section{Méthodes palÉOdosimétriqueS}

Les méthodes dites paléodosimétriques recouvrent un ensemble de méthodes de datation basées sur l'intégration d'énergie au cours du temps issue des interactions des rayonnements ionisants et des minéraux. Dans cette catégorie de méthodes, nous trouvons la luminescence avec ses différentes variantes (thermoluminescence $-\mathrm{TL}-$, luminescence optiquement stimulée - OSL - ou stimulée par infra-rouge 
- IRSL -, radiofluorescence - RF - etc.) et la résonance paramagnétique électronique (RPE ou ESR " electron spin resonance " en anglais). Toutes ces méthodes reposent sur le principe d'un instant zéro, qui est l'instant d'intérêt représentant le démarrage ou le redémarrage du fonctionnement du dosimètre naturel. Ce peut-être le chauffage ancien, ou la fin de l'exposition à la lumière ou encore la formation du minéral (dans la mesure où cette formation n'entraîne pas un état initial distinct de zéro). Le moteur de ces méthodes est la radioactivité naturelle (en supposant négligeable la contribution d'événements nucléaires artificiels localisés). Ainsi du point de vue dosimétrique, c'est-à-dire peu ou prou de la quantité d'énergie émise, le potassium, l'uranium, le thorium et marginalement le rubidium représentent la totalité des éléments-sources de rayonnement ionisants «terrestres » (Guérin et al., 2011 pour une mise à jour récente des correspondances entre débit d'énergie, nature des rayonnements et concentration des radio-isotopes naturels). À cette radioactivité liée aux séries naturelles s'ajoute la contribution du rayonnement cosmique qui dépend de l'altitude, de la profondeur d'enfouissement ou des effets d'écran de l'environnement de l'objet daté, et aussi dans une moindre mesure de la latitude du site (Prescott et Hutton, 1994). Du point de vue du récepteur, c'est-à-dire du matériau dosimètre, celui qui sera soumis à la datation, le processus d'enregistrement des interactions avec les rayonnements ionisants est justement l'ionisation des atomes. Pour une interaction donnée, il y aura formation de paires électron-trou mobiles dans le cristal proportionnellement à l'énergie cédée par une particule ou un photon gamma. La grande majorité des paires formées se recombine, cependant la capture d'électrons ou de trous au niveau de défauts ponctuels ou d'impuretés à l'échelle atomique dans le cristal est effective et est à l'origine de l'enregistrement dosimétrique. Le nombre de pièges ayant capturé des charges mobiles croît avec l'irradiation et donc avec le temps, jusqu'à saturation du système (Aitken, 1985). Le chauffage du matériau irradié ou son éclairement entraine la libération des paires électron - trou piégées. Ce processus est généralement accompagné de luminescence.

Toutes ces méthodes, du point de vue pratique, répondent à la même équation d'âge qui consiste au rapport de la dose archéologique à la dose annuelle. La dose est la quantité d'énergie absorbée par un matériau par unité de masse et plus précisément ici, il s'agit de la dose accumulée depuis l'instant archéologique d'intérêt, d'où l'expression " dose archéologique ». La dose annuelle est le débit annuel de dose absorbée par le matériau daté. On la suppose constante dans le temps en raison des demi-vies des radioéléments parents qui avoisinent ou dépassent largement le milliard d'années, cependant il est nécessaire de s’assurer de cette permanence de la dose annuelle, en raison de possibles altérations de nature chimique des matériaux au cours de l'enfouissement, ou de modifications de leur environnement gamma et cosmique. On parle aujourd'hui de dose annuelle moyenne. Toute la problématique des méthodes paléodosimétriques se " résume " à déterminer dose archéologique et dose annuelle de façon précise et juste.

Durant les quatre dernières décennies, la datation par luminescence, méthode sur laquelle je vais à présent insister, a connu et connaît des développements très importants, tant au niveau des concepts, qu'au niveau instrumental et de la mise en œuvre.

La thermoluminescence était utilisée dans les années 1950 comme méthode d'appoint de caractérisation des minéraux. Les géologues ont alors constaté que d'une manière statistique la luminescence thermostimulée pour un type de roche déterminé était d'autant plus intense que la roche était ancienne. Les premières applications en tant que méthode de datation reviennent aux travaux de pionniers des groupes d'Oxford (Aitken et al., 1964) et de Risoe au Danemark (Mejdahl, 1969) sur des céramiques. C'est dans les années 1970 que se sont installés en France, les laboratoires de Bordeaux, Gif-sur-Yvette et Clermont-Ferrand. En 1977, le paysage français du microcosme de la datation par thermoluminescence est en place ou presque (le C2RMF s'équipera quelques années plus tard). L'application majeure à cette époque concerne la datation par thermoluminescence de matériaux anciennement chauffés (céramiques, pierres de foyer, silex...). Le laboratoire de Risoe tend à se spécialiser dans la datation du blanchiment optique des quartz et des feldspaths par thermoluminescence.

Une première révolution est arrivée lorsque la luminescence optiquement stimulée est sortie des domaines de la dosimétrie des irradiations en milieu nucléaire et médical, et de l'imagerie infra-rouge et a été appliquée à la datation de l'enfouissement de sédiments (Huntley et al., 1985). On montre alors que l'OSL ou l'IRSL est bien plus efficace que la TL pour cette application car la remise à zéro du signal d'OSL ou d'IRSL est totale ou presque par opposition à la TL où un important signal résiduel demeure. La porte est désormais ouverte aux études paléoenvironnementales et un important développement pour la datation des sédiments éoliens, de sables dunaires, s'en suit au niveau international. Les recherches méthodologiques s'amplifient pour comprendre la nature des signaux d'OSL et isoler les composantes les plus intéressantes pour la datation. Ainsi à la différence de la thermoluminescence où la rampe de chauffage depuis l'ambiante jusque vers $500{ }^{\circ} \mathrm{C}$ permet de séparer les émissions selon leur stabilité thermique et donc leur aptitude à la datation, l'OSL permet de séparer les émis- 
sions selon une sensibilité décroissante à l'éclairement et il n'y pas de relation univoque avec la stabilité thermique, si ce n'est que l'énergie du photon d'excitation doit être supérieure à l'énergie d'activation requise pour libérer les charges piégées (la composante dite rapide de l'OSL des quartz est suffisamment stable thermiquement, alors que la composante dite moyenne ne l'est pas). La méthode devient véritablement opérationnelle dans le courant des années 1990, avec la mise au point d'appareils spécifiques, la mise en place de protocoles de mesure standardisés (Murray et Wintle, 2000). Cependant les tentatives pour dater les dépôts de sédiments fluviatiles ou déposés par ruissellement échouent ou tout au moins suscitent un certain degré de circonspection. Tous les dépôts sédimentaires ne sont pas datables car des cas d'exposition insuffisante à la lumière sont mis en évidence, de même que des sédiments où des perturbations post-dépositionnelles sont apparues. La communauté réfléchit alors à la façon de résoudre ce type de problème. La solution apparaît dans l'étude monograin (« single grain OSL dating»: SG-OSL), où l'examen de grains individuels doit permettre de séparer les grains bien " blanchis » des autres. C'est une seconde révolution dans le monde de la datation par luminescence qui prend place et dont nous n'avons pas encore terminé d'en mesurer les conséquences. C'est un changement d'échelle de travail qu'il faut à présent prendre en compte avec l'étude de plusieurs milliers de grains par échantillon. On ne raisonne plus sur une valeur unique de la dose archéologique, mais sur une distribution de doses. On ne raisonne plus non plus sur une valeur unique de la dose annuelle mais sur une distribution à l'échelle des grains.

Après une première phase de réflexion sur le traitement des données et de proposition à la fin des années 1990 (Galbraith et al., 1999), le travail théorique sur de nouveaux modèles statistiques plus adaptés à la physique des grains et des modalités de dépouillement continue aujourd'hui de se développer et même si les nouveaux outils ne sont pas encore complètement stabilisés, une bien meilleure compréhension des processus physiques, selon mon point de vue, est à portée de main (Christophe et al., 2018; Guérin et al., 2017; Guibert et al., 2017). Aujourd'hui, le gain qualitatif, c'est aussi la capacité de décider par des considérations intrinsèques à la méthode, à la vue de l'histogramme de distribution des doses, si l'échantillon a été globalement bien blanchi ou non et si la datation que nous pouvons proposer sera valide ou susceptible de "révision majeure ".

Ces quelques lignes sur le développement de la datation par luminescence ne sauraient occulter un travail méthodologique de longue haleine permanent des chercheurs par exemple sur la question de la prise en compte des déséquilibres dans les séries de l'uranium, signature de processus d'altération. Il en va de même sur le travail récurrent de modélisation des irradiations dans des environnements complexes, multi-scalaires et hétérogènes (Martin et al., 2015) qui s'est concrétisé grâce aux capacités de calcul disponibles depuis quelques années. Il n'est pas question de rentrer davantage dans un exposé détaillé de l'évolution de la datation par luminescence, il faut cependant avoir à l'esprit que la datation par des méthodes paléodosimétriques, pour la maîtriser, requiert des connaissances théoriques et pratiques multidisciplinaires en physique du solide, physique nucléaire, physique instrumentale, géochimie, sciences archéologiques, qui échappent de plus en plus au chercheur isolé. Il s'agit bien du travail interdisciplinaire d'un groupe.

Dans le domaine de l'archéologie, la datation par luminescence dont la portée s'étend jusque vers 300ka s'est beaucoup développée pour la chronologie des cultures paléolithiques, notamment celles du Paléolithique Moyen, pour les périodes que le radiocarbone ne permet plus de dater au-delà de $45 \mathrm{ka}$ BP (par ex Valladas et al., 1999; Mercier et al., 1991, 2007; Guérin et al., 2012; Tribolo et al., 2009). L'extension de la portée de la méthode jusqu'au million d'années reste un objectif éloigné en raison de l'effet de saturation des matériaux malgré les espoirs fondés sur toute nouvelle technique mise en œuvre. En revanche, la place de la luminescence, en complémentarité des autres méthodes est totalement justifiée pour les périodes plus récentes. On citera par exemple les travaux de C. Lahaye sur le peuplement ancien du Brésil, significativement plus ancien que ce que la communauté des américanistes supposait (Lahaye et al., 2013). Nous citerons également nos propres travaux en collaboration avec nos collègues de Milan, Durham et Catane sur les constructions du haut Moyen Âge (Guibert et al., 2012) ainsi que la mise au point de la datation des mortiers de chaux par SG-OSL (Urbanová et Guibert, 2017) et l'ouverture vers l'archéologie du bâti.

La datation par luminescence permet à présent de travailler sur un large spectre thématique pour toute période entre les premières manifestations des cultures moustériennes et l'actuel. Elle n'est plus réservée aux seuls objets chauffés comme on l'entend encore quelques fois mais s'adresse à de très nombreuses situations où il s'agit de dater une chauffe ou une mise au jour ancienne.

La RPE que nous n'avons que très peu évoquée est minoritaire dans la communauté paléodosimétrique (en France, elle repose sur 3 permanents chercheurs spécialistes). Pourtant, cette méthode offre de nombreuses applications sur émail dentaire fossile et de très nombreux sites paléolithiques en Europe ont pu bénéficier de telles datations (Falguères, 2003). Signalons que l'équipe du MNHN avec une équipe japonaise a mis au point une méthode de datation de dépôts 
sédimentaires quaternaires (Toyoda et al., 2000 ; Tissoux et al., 2008) basée sur la photosensibilité de centres $\mathrm{Al}$ et Ti dans le quartz, rejoignant ici les préoccupations de la communauté des spécialistes d'OSL. L'avantage de la RPE par rapport à la luminescence est la capacité d'identifier les centres paramagnétiques, car il s'agit d'une spectrométrie, alors que le signal d'OSL est global et non "signé ». Un autre avantage de la RPE est la possibilité d'accéder à des âges très supérieurs à la luminescence, au-delà du million d'années, car la saturation des centres paramagnétiques avec l'irradiation n'intervient qu'à de très fortes doses (voir par exemple les résultats de datation de dépôts sédimentaires de la moyenne vallée de la Loire : Voinchet et al., 2010).

\section{Conclusion}

Pour tenter de conclure cet état des lieux sur l'évolution des méthodes de datation pour l'archéologie, on notera que les progrès majeurs, dans le sens d'un accroissement des connaissances et du potentiel d'acquisition de nouvelles connaissances, sont très étroitement liés à l'évolution des techniques de mesure et du traitement de l'information. Ainsi, la sensibilité extraordinaire atteinte par les spectromètres de masse a permis de développer considérablement la datation par les isotopes cosmogéniques, radiocarbone compris, mais aussi pour les couples de radioéléments K-Ar ou ${ }^{234} \mathrm{U}_{-}{ }^{230} \mathrm{Th}$, pour reprendre les méthodes présentées ici. La diminution de la taille requise de l'échantillon, ou l'extrême sensibilité à des éléments en traces ont ouvert de nouvelles possibilités de datation et de manière de travailler. Pour la luminescence, c'est la mise en place de l'OSL qui a modifié profondément le paysage de la recherche, puis le développement des techniques monograin qui aujourd'hui renouvellent la méthode. En clair, de nouveaux défis sont en cours pour la chronologie et c'est une chance que les chercheurs doivent saisir.

L'opération ultime de la datation, c'est la modélisation chronologique, qui a pour objectif d'intégrer les données de la chronologie physique et les données de chronologie relative déduites des observations stratigraphiques et archéologiques. Le développement de méthodes de traitement des données et de logiciels de calcul correspondants ont pour objectif de proposer in fine des intervalles chronologiques affinés prenant en compte toutes les données tant quantitatives que qualitatives. Nous citerons à cette occasion, la méthode et le logiciel CHRONOMODEL(C) (Lanos et Philippe, 2017) qui se distingue d'autres systèmes comme OxCal par le traitement des singularités et des anomalies de datation (gestion des " outliers") en partant du principe que les incertitudes de datation fournies par les spécialistes sont des incertitudes minimales et que des facteurs dispersifs non implicites liés par exemple à la nature du site sont possibles. Cette modélisation c'est-à-dire l'opération de mise en place des diagrammes chronologiques, en prévision des calculs statistiques, induit une nouvelle stratégie de datation et de prise d'échantillons. Il est ainsi largement préférable de dater une séquence entière encadrant les niveaux d'intérêt à dater plutôt que de se limiter strictement à ceux-ci. La raison est que la stratigraphie ou plus généralement la chronologie relative des événements, impose une contrainte probabiliste aux résultats de datation, réduisant ainsi de proche en proche les intervalles d'incertitude. Il est aussi conseillé, de longue date d'ailleurs, d'accroître le nombre d'échantillons datés, non pas pour satisfaire un trivial intérêt économique, ni donner plus de travail aux dateurs, mais pour contrôler les effets dispersifs potentiels et accroittre la précision statistique. Le summum serait d'appliquer plusieurs méthodes distinctes, pour un même événement afin de limiter les effets des incertitudes systématiques propres à une méthode, une technique et une pratique de laboratoire.

La recherche de la qualité de la datation, c'est-à-dire, la mise en œuvre effective des procédures et protocoles justifiées par un objectif de précision et de justesse et leur publication, doit rester l'objectif du chronologue, c'est cette évidence que je voudrais rappeler ici. Les budgets contraints, les échéances « intenables ", les difficultés croissantes de financement de la recherche agissent bien souvent au détriment de la qualité des résultats, y compris même au sein de notre écosystème scientifique, alors que nous venons de clamer la nécessité de multiplier l'échantillonnage dans les lignes qui précèdent et d'accroître l'ambition chronologique de nos études!

Il est ainsi nécessaire d'assurer la pérennité des compétences sur le long terme à défaut d'être capable d'accroître notre potentiel de recherche. La datation est une activité scientifique et technique de haut niveau qui, la plupart du temps, passe relativement inaperçue tant elle est évidente, permanente, besogneuse, à l'écart des effets d'annonce. Elle engage le chronologue et ses équipes sur le long terme. La datation est indispensable à la caractérisation et à l'interprétation des faits archéologiques, et je me permets ici d'enfoncer le clou une nouvelle fois sur l'importance de cette problématique auprès des établissements de recherche concernés pour rappeler le devoir de recrutement de chercheurs et d'ingénieurs de haut niveau sur cette thématique. Tel est sans doute le plus grand défi pour la datation. L'urgence est permanente car nos systèmes restent trop fragiles. 


\section{Remerciements}

Mes remerciements institutionnels vont au réseau CAI-RN (Compétences Archéométriques Interdisciplinaires - Réseau National, sous les auspices de la mission pour l'interdisciplinarité du CNRS) et au GMPCA (Groupe des Méthodes Pluridisciplinaires Contribuant à l'Archéologie, association loi 1901) sans lesquels ni la conférence au colloque de Rennes d'avril 2017, ni cet article n'auraient pu exister, et à mon établissement, le CNRS, qui verse mon salaire. J'adresse mes remerciements les plus chaleureux aux collègues que j'ai interrogés et qui ont bien voulu répondre à mes questions, me transmettre des éléments de bibliographie, me parler de leurs problématiques et pour certains relire le manuscrit. J'espère ne pas avoir trahi leur pensée. Il sagit de Vincent Bernard (CNRS, UMR CREAAH à Rennes), Philippe Lanos (CNRS, IRAMAT-CRP2A à Géosciences Rennes), Gwénaël Hervé (post-doctorant au CEREGE à Aix), Christophe Moreau et Emmanuelle DelquéKolič (CNRS, UMR LSCE, LMC14 à Gif-sur-Yvette), Didier Bourlès (CNRS, CEREGE à Aix) qui m'a prêté ses cours il y a quelques années et que j'ai de nouveau sollicité ici, Véronique Michel (CNRS, CEPAM à Nice), Jean-Claude Lefevre (CNRS, laboratoire de datation par le radiocarbone, UMR ArAr à Lyon), Chantal Tribolo (CNRS, IRAMAT-CRP2A) avec tous mes collègues du groupe luminescence de Bordeaux. Je remercie aussi Ludovic Bellot-Gurlet pour sa patience.

\section{Bbliographie}

Aitken M.J., 1985. Thermoluminescence dating. Academic Press, London, $359 \mathrm{p}$.

Aitken M.J., Tite M.S., Reid J., 1964. TL-dating of ancient pottery. Nature, 202, p. 1032.

Aldrich L.T., Nier A.O., 1948. Argon 40 in potassium minerals. Physical Review, 74, p. 876-877.

Astrade L., Miramont C., 2010. Panorama de la Dendrochronologie en France, coll. "Edytem ", no 11, 210 p., ISBN : 978-2-918435-03-7; [https://hal.archives-ouvertes.fr/ halsde-00786410].

Bard E., Ménot G., Rostek F., Licari L., Böning P., Edwards R.L., Cheng H., Wang Y.J., Heaton T.J., 2013. Radiocarbon calibration/comparison records based on marine sediments from the Pakistan and Iberian Margins. Radiocarbon, 55 (4), p. 1999-2019.

Bard E., Tuna T., Fagault Y., Bonvalot L., Wacker L., Fahrni S., Synal H.A, 2015. AixMICADAS, the accelerator mass spectrometer dedicated to ${ }^{14} \mathrm{C}$ recently installed in Aix-enProvence, France. Nuclear Instruments and Methods in Physics Research Section B, 361, p. 80-86.
Barrett G.T., 2017. Rehydroxylation (RHX) dating: Mass loss issues due to incomplete drying, carbon content, and mineral alteration. Journal of Archaeological Science, Reports, 16, p. 472488.

Bernard V., Langouet L., 2014. Early Middle Ages Fishweirs, Dendrochronology and Wood Supply in Western France: The Case of the Léguer Estuary, Servel-Lannion, Northern Brittany, France. Journal of Wetland Archaeology, 14-1, p. 34-47.

Billamboz A., 2014. Timber from Old and Young Trees: Dendrotypology as the Backbone of the Dendroarchaeological Investigations of Prehistoric Fish Traps and Pile Dwellings in South-West Germany. Journal of Wetland Archaeology, 14-1, p. $48-57$.

Blackwell B., Schwarcz H.P., Debénath A., 1983. Absolute dating of Hominids and Paleolithic artifacts of the cave of La Chaise-de-Vouthon (Charente), France. Journal of Archaeological Science, 10, p. 493-513.

Bourlès D., Raisbeck G.M., Yiou F., 1989. ${ }^{10} \mathrm{Be}$ and ${ }^{9} \mathrm{Be}$ in marine sediments and their potential for dating. Geochimica et Cosmochimica Acta, 53, p. 443-452.

Brown A.T., Edmond J.M., Raisbeck G.M., Yiou F., Kurz M.D., Brook E.J., 1991. Examination of surface exposure ages of Antarctic moraines using in situ produced ${ }^{10} \mathrm{Be}$ and ${ }^{26}$ Al. Geochimica et Cosmochimica Acta, 55, p. 2269-2283.

Christophe C., Philippe A., Guérin G., Mercier N., Guibert P., 2018. Bayesian approach to OSL dating of poorly bleached sediment samples: Mixture Distribution Models for Dose $\left(\mathrm{MD}^{2}\right)$. Radiation Measurements, 108, p. 59-73.

Couchoud I., Genty D., Hoffmann D., Drysdale R., Blamart D., 2009. Millennial-scale climate variability during the Last Interglacial recorded in a speleothem from south-western France. Quaternary Science Reviews, 28, p. 3263-3274.

Deino A.L., Renne P.R., Swicher C.C., 1998. ${ }^{40} \mathrm{Ar} /{ }^{39} \mathrm{Ar}$ in Paleoanthropology and Archaeology. Evolutionary Anthropology: Issues, News and Reviews, 6(2), p. 63-75.

Ericson J.E., Dersch O., Rauch F., 2004. Quartz hydration dating. Journal of Archaeological Science, 31, p. 883-902.

FAlguères C., 2003. ESR dating and the human evolution: contribution to the chronology of the earliest humans in Europe. Quaternary Science Reviews, 22, p. 1345-1351.

Friedrich M., Remmele S., Kromer K., Hofmann J., Spurk M., Kaiser K.F., Orcel C., Küppers M., 2004. The 12,460 year Hohenheim oak abd pine tree ring chronology from central Europe - A unique annual record for radiocarbon calibration and paleoenvironment reconstructions. Radiocarbon, 46(3), p. 1111-1122.

Galbraith R.F., Roberts R.G., Laslett G.M., Yoshida H., OlleY J.M., 1999. Optical dating of single and multiple grains of quartz from Jinmium Rock Shelter, Northern Australia: part 
I, experimental design and statistical models. Archaeometry, 41(2), p. 339-364.

Gallet Y., Geneveyn A., Le Goff M., Warmé N., GranAymerich J., Lefèvre A., 2009. On the use of archeology in geomagnetism, and vice-versa: Recent developments in archeomagnetism. Comptes Rendus Physique, 10(7), p. 630-648.

Gascoyne M., 1982. Geochemistry of the actinides and their daughters. In Uranium series disequilibrium: applications to environmental problems, Ivanovitch and Harmon Ed., Clarendon Press, Oxford, p. 33-55.

Genevey A., Gallet Y., Jesset S., Thébault E., Bouillon J., Lefèvre A., Le Goff M., 2016. New archeointensity data from French Early Medieval pottery production (6th-10th century AD). Tracing 1500 years of geomagnetic field intensity variations in Western Europe. Physics of the Earth and Planetary Interiors, 257, p. 205-219.

Genevey A., Gallet Y., Thébault E., Jesset S., Le Goff M., 2013. Geomagnetic field intensity variations in Western Europe over the past 1100 years. Geochemistry, Geophysics, Geosystems, 14, p. 2858-2872.

Genty D., Ghaleb B., Plagnes V., Causse C.H., Blamart D., Massault M., Geneste J.M., Clottes J., 2004. Datations U/ Th (TIMS) et 14C (AMS) des stalagmites de la grotte Chauvet (Ardèche, France) : intérêt pour la chronologie des événements naturels et anthropiques de la grotte. Comptes Rendus Palevol, 3, p. 629-642.

Ghaleb B., Falguères C., 2017. Apport des méthodes basées sur le déséquilibre radioactif $\left({ }^{238} \mathrm{U}-{ }^{234} \mathrm{U}-{ }^{230} \mathrm{Th}-{ }^{226} \mathrm{Ra}-{ }^{210} \mathrm{~Pb}\right)$ aux études des variations et changements climatiques. L'Anthropologie, 121, p. 73-81.

Gillot P-Y., Chiesa S., Pasquaré G., Vezzoli L., 1982. <33,000 years $\mathrm{K}$-Ar dating of the volcano-tectonic horst of the Isle of Ischia, Gulf of Naples. Nature, 299, p. 242-244.

Gómez-Paccard M., Chauvin A., Lanos Ph., Dufresne Ph., Kovacheva M., Hill M.J., Beamud E., Blain S., Bouvier A., Guibert P., 2012. Improving our knowledge of rapid geomagnetic field intensity changes observed in Europe between 200 and 1400 AD. Earth and Planetary Science Letters, 355356, p. 131-143.

Gómez-Paccard M., Osete M.L., Chauvin A., Pavón-Carrasco F.J., Pérez-Asensio M., Jiménez P., Lanos Ph., 2016. New constraints on the most significant paleointensity change in Western Europe over the last two millennia. A non-dipolar origin? Earth and Planetary Science Letters, 454, p. 55-64.

Goulpeau L., Lanos Ph., Langouët L., 1989. The anisotropy as a disturbance of the archaeomagnetic dating method. In Archaeometry, Proceedings of the $25^{\text {th }}$ International Symposium, Elsevier Publishers, p. 45-58.

Guérin G., Combès B., lahaye C., Thomsen K.J., Tribolo C., Urbanová P., Guibert P., Mercier N., Valladas H.,
2015. Testing the accuracy of a Bayesian central-dose model for single-grain OSL, using known-age samples. Radiation Measurements, 81, p. 62-70.

Guérin G., Mercier N., Adamiec G., 2011. Dose-rate conversion factors: update. Ancient TL, 29 (1), p. 5-8.

Guibal F., 1992. First dendrochronological dating of a shipwreck in the western Mediterranean area. Dendrochronologia, 10, p. 147-156.

Guibert P., Brodard A., Quilès A., Geneste J.-M., Baffier D., Debard E., Ferrier C., 2015. When were the walls of the Chauvet Pont-d'Arc Cave heated? A chronological approach by thermoluminescence. Quaternary Geochronology, 29, p. 36-47.

Guibert P., Christophe C., Urbanová P., Blain S., Guérin G., 2017. Modeling incomplete and heterogeneous bleaching of mobile grains partially exposed to the light: towards a new tool for single grain OSL dating of poorly bleached mortars, Radiation Measurements, 107, p. 48-57.

Gutiérrez Garcia-Moreno A., 2014. La producción de material lapídeo en el norte del conventus Tarraconensis : extracción, organización y gestión de las canteras. In J. Bonetto, S. Camporeale, A. Pizzo (éd.), Arqueología de la Construcción IV. Le cave nel mondo antico : sistemi di sfruttamento e processi produttivi, Anejos de Archivo Español de Arqueología, Madrid-Mérida, Madrid, p. 311-328.

Hajdas I., Lindroos A., Heinemeier J., Ringbom Å., Marzaioli F., Terrasi F., Passariello I., Capano M., Artioli G., Addis A., Secco S., Michalska D., Czernik J., Goslar T., Hayen R., Van Strydonck M., Fontaine L., Boudin M., Maspero F., Panzeri L., Galli A., Urbanová P., Guibert P., 2017. Preparation and dating of mortar samples - Mortar Dating Inter-comparison Study (MODIS). Radiocarbon, 56(6), p. 1845-1858..

Hervé G., Lanos Ph., 2013. Datation archéomagnétique : contribution du champ géomagnétique à l'archéologie. Spectra Analyse, 292, p. 39-45.

Hervé G., Chauvin A., Lanos Ph., 2013a. Geomagnetic field variations in Western Europe from 1500 BC to 200 AD. Part I: Directional secular variation curve. Physics of the Earth and Planetary Interiors, 218, p. 1-13.

Hervé G., Chauvin A., Lanos Ph., 2013b. Geomagnetic field variations in Western Europe from 1500 BC to 200 AD. Part II : New intensity secular variation curve. Physics of the Earth and Planetary Interiors, 218, p. 51-65.

Hervé G., Fassbinder J., Gilder S., Metzner-Nebelsick C., Gallet Y., Genevey A., Schnepr E., et al., 2017. Fast geomagnetic field intensity variations between 1400 and 400 BCE: New archaeointensity data from Germany. Physics of the Earth and Planetary Interiors, 270, p. 143-156.

Hoffsummer P., 2002. Les charpentes du XI au XIX siècle: typologie et évolution en Belgique du Nord et en Belgique. Paris, MonumÉditions du Patrimoine, coll. « cahiers du Patrimoine; 62 », 376 p. 
Hoffsummer P., 1999. Les charpentes. In A. Dierkens (dir.), L'ancienne église abbatiale de Saint-Hubert. Namur, Études et Documents, "Monuments et Sites; 7 », p. 90-95.

Huntley D.J., Godfrey-Smith D.I., Thewalt M.L.W., 1985. Optical dating of sediments. Nature, 313, p. 105-107.

Labeyrie J., Delibrias G., 1964. Dating of old mortars by the carbon-14 method. Nature, 201, p. 742.

Lahaye C., Hernandez M., Boëda E., Daltrini Felice G., Guidon N., Hoeltz S., Lourdeau A., Pagli M., Pessis A.-M., Rasse M., Viana S., 2013. Human occupation in South America by 20,000 BC: the Toca da Tira Peia site, Piauí, Brazil. Journal of Archaeological Science, 40, p. 2840-2847.

LAmbert G., Lavier C., 1990. Dendrochronologie et préhistoire. Bulletin de la Société préhistorique française, 87 (5), p. 143-152.

LANOs Ph., 1987. The effects of demagnetizing fields on thermoremanent magnetization acquired by parallel-sided baked clay blocks. Geophysical Journal of Royal Astronomical Society, 91, p. 985-1012.

Lanos Ph., 1990. La datation archéomagnétique des matériaux de construction d'argile cuite, apports chronologiques et technologiques. Gallia, 47, p. 321-341.

Lanos Ph., 1994. Pratiques artisanales des briquetiers et archéomagnétisme des matériaux d'argile cuite. Une histoire de positions de cuisson. Histoire \& Mesure, IX - 3/4, p. 287-304.

Lanos Ph., Kovacheva M., Chauvin A., 1999. Archaeomagnetism, methodology and applications: implementation and practice of the archaeomagnetic method in France and Bulgaria. European Journal of Archaeology, 2(3), p. 365-392.

Lanos Ph., 2004. Bayesian inference of calibration curves, application to archaeomagnetism: Chapter 3 in "Tools for Constructing Chronologies, Crossing Disciplinary Boundaries". Series: Lecture Notes in Statistics, vol. 177, Buck C.E. and Millard A.R. (eds), London, Springer-Verlag, p. 43-82.

Lanos Ph., Philippe A., 2017. Hierarchical Bayesian modeling for combining dates in archaeological context. Journal de la Société Française de Statistique, 158 (2), p. 72-88.

lanphere M., Champion D., Melluso L., Morra V., Perrotta, A., Scarpati C., Tedesco D., Calvert A., 2007. ${ }^{40} \mathrm{Ar} /{ }^{39} \mathrm{Ar}$ ages of the AD 79 eruption of Vesuvius, Italy. Bulletin of Volcanology, 69, p. 259-263.

Lebatard A.E., Bourlès D., Duringer P., Jolivet M., Braucher R., Carcaillet J., Schuster M., Arnaud N., Monié P., Lihoreau F., Likius A., Mackaye H.T., Vignaud P., Brunet M., 2008. Cosmogenic nuclide dating of Sahelanthropus tchadensis and Australopithecus bahrelghazali: Mio-Pliocene hominids from Chad. PNAS, 105(9), p. 3226-3231.

Lefèvre J.-Cl., Gillot P.-Y., 1994. Datation Potassium-Argon de roches volcaniques du Pléistocènes supérieur et de l'Holocène,
Exemple de l'Italie du Sud; application à l'archéologie. Bulletin de la Société Préhistorique Française, 91 (2), p. 145-148.

Leroy S., L'Héritier M., Delqué-Količ E., Dumoulin J.-P., Moreau C., Dillmann P., 2015. Consolidation or initial design? Radiocarbon dating of ancient iron alloys sheds light on the reinforcements of French Gothic Cathedrals. Journal of Archaeological Science, 53, p. 190-201.

Libby W.F., Anderson E.C., Arnold J.R., 1949. Age determination by radiocarbon content: World-wide assay of natural radiocarbon, Science, 109 (2827), p. 227-228.

Maggi C., Pagès G., Mertens A., Hoffsummer P., 2012. Utilisation et technique de production du fer et du bois dans les charpentes de comble mosanes : premiers jalons d'une évolution du XII ${ }^{\mathrm{e}}$ au XVIII ${ }^{\mathrm{e}}$ siècle, ArcheoSciences-Revue d'Archéométrie, 36, p. 95-115.

Martin L., Mercier N., Incerti S., Lefrais Y., Pecheyran C., Guérin G., Jarry M., Bruxelles L., Bon F., Pallier C., 2015. Dosimetric study of sediments at the beta dose rate scale: Characterization and modelization with the DosiVox software. Radiation Measurements, 81, p. 134-141.

Mc Dougall I., 2014. K/Ar and ${ }^{40} \mathrm{Ar} /{ }^{39} \mathrm{Ar}$ Isotopic Dating Techniques as Applied to Young Volcanic Rocks, Particularly Those Associated with Hominin Localities. in A. M. Davis Ed., Treatise on Geochemistry, $2^{\text {nd }}$ Edition, p. 1-15.

Mejdahl V., 1969. Thermoluminescence dating of ancient danish ceramics. Archaeometry, 11, p. 99-104.

Mercier N., Valladas H., Froget L., Joron J.-L., Reyss J.L., Weiner S., Goldberg P., Meignen L., Bar-Yosef O., Belfer-Cohen A., Chech M., Kuhn S.L., Stiner M.C., Tillier A.-M., Arensburg B., Vandermeersch B., 2007. Hayonim Cave: A TL-based chronology for this Levantine Mousterian sequence. Journal of Archaeological Science, 34, p. 1064-1077.

Mercier N., Valladas H., Joron J.-L., Reyss J.-L., Lévêque F., Vandermeersch B. 1991. Thermoluminescence dating of the late Neanderthal remains from Saint-Césaire. Nature, 351, p. 737-739.

Mercier N., Kreutzer S., Christophe C., Guérin G., Guibert P., Lahaye C., Lanos Ph., Philippe A., Tribolo C., 2016. Bayesian statistics in luminescence dating: The "baSAR"-model and its implementation in the $\mathrm{R}$ package "Luminescence". Ancient TL, 34, p. 14-21.

Michel V., Shen G., Shen C.C., Wu C.C., Vérati C., Gallet S., Moncel M.H., Combier J., Кнatib S., Manetti M., 2013. Application of U/Th and ${ }^{40} \mathrm{Ar} /{ }^{39} \mathrm{Ar}$ Dating to Orgnac 3, a Late Acheulean and Early Middle Palaeolithic Site in Ardèche, France. PLOS ONE, 8(12), e82394.

Millard A. R., Hedges R. E. M., 1996. A diffusion-absorption model of uranium uptake by archaeological bone. Geochimica et Cosmochimica Acta, 60 (12), p. 2139-2152. 
Miramont C., Guibal F., Kaiser K.F., Kromer B., Sgier M., Sivan O., Friedrich M., Talamo S., 2010. L'apport des séries dendrochronologiques françaises au prolongement de la chronologie européenne absolue et à la calibration du radiocarbone. in Laurent Astrade et Cécile Miramont (dir.), Panorama de la dendrochronologie en France, Edytem, no 11, p. 181-190.

Muller R.A., 1977. Radio-isotope dating with a cyclotron. Science, 196 (4289), p. 489-494.

Murray A.S., Wintle A.G., 2000. Luminescence dating of quartz using an improved single-aliquot regenerative-dose protocol. Radiation Measurements, 32, p. 57-73.

Pécheyran C., Claverie F., Barbotin G., Donard O., Pointurier F., Grijalba N., Unceta N., Martin L., Tribolo C., Mercier N., 2016. Ultra trace and isotopic analysis by high repetition rate femtosecond laser ablation coupled to ICPMS. $9^{\text {th }}$ International Conference on Laser-Induced Breakdown Spectroscopy (LIBS), september 12-16, Chamonix, France.

Pécheyran C., Claverie F., Tabouret V., Bareille G., Grijalba N., Unceta N., Bérail S., Mercier N., Martin L., Tribolo C., Daverat F., Garnier D., 2017. Lablation Laser ICPMS : de l'élémentaire à l'isotopie, de l'environnement à la lutte contre la contrefaçon, des premières expressions artistiques de l'homme moderne à la poésie contemporaine, Journée IPGP consacrée aux développements de la mesure Isotopique, 28 avril 2017, Paris, France.

PÈtrequin P., 2005. Habitats lacustres néolithiques et perception du temps, Bulletin de la Société préhistorique française, 102 (4), p. 789-802.

Petтitт P., 2008. Art and the Middle-to-Upper Paleolithic transition in Europe: Comments on the archaeological arguments for an early Upper Paleolithic antiquity of the Grotte Chauvet art. Journal of Human Evolution, 55, p. 908-917.

Pons-Branchu E., Douville E., Roy-Barman M., Dumont E., Branchu P., Thil F., Frank N., Bordier L., Borst W., 2014. A geochemical perspective on Parisian urban history based on U-Th dating, laminae counting and yttrium and REE concentrations of recent carbonates in underground aqueducts. Quaternary Geochronology, 24, p. 44-53.

Prescott J.R., Hutton J.T., 1994. Cosmic ray contributions to dose rates for luminescence and ESR dating: large depths and long-term time variations. Radiation Measurements, 23 (2/3), p. 197-200.

Quilès A., Valladas H., Bocherens H., Delqué- Količ E., Kaltnecker E., van der Plicht J., Delannoy J.J., Feruglio V., Fritz C., Monney J., Philippe M., Tosello, G., Clottes J., Geneste J.M., 2016. A high-precision chronological model for the decorated Upper Paleolithic cave of Chauvet-Pont d'Arc, Ardèche, France. PNAS, 113 (17), p. 4670-4675.

Quilès A., Valladas H., Geneste J.M., Clottes J., Baffier D., Berthier B., Brock F., Bronk Ramsey C., DelquéKolič E., Dumoulin J.P., Hajdas I., Hippe K., Hodgins
G.W.L., Hogg A., Jull A.J.T., Kaltnecker E., de Martino M., Oberlin C., Petchey F., Steier P., Synal H.A., van der Plicht J., Wild E.M., Zazzo A., 2014. Second Intercomparison Program for the Chauvet Pontd'Arc Cave Ardèche France. Radiocarbon, 56(2), p. 833850.

Quinif Y., Genty D., Maire R., 1994. Les spéléothèmes : un outil performant pour les études paléoclimatiques. Bulletin de la Société Géologique de France, 165 (6), p. 603-612.

Reimer P.J., Bard E., Bayliss A., Beck J.W., Blackwell P.G., Bronk Ramsey C., Grootes P.M., Guilderson T.P., Haflidason H., Hajdas I., Hatté C., Heaton T.J., Hoffmann D.L., Hogg A.G., Hughen K.A., Kaiser K.F., Kromer B., Manning S.W., Niu M., Reimer R.W., Richards D.A., Scott E.M., Southon J.R., Staff R. A., Turney C. S.M., van Der Plicht J., 2013. IntCal13 and Marine13 Radiocarbon Age Calibration Curves 0-50,000 Years cal BP. Radiocarbon, 55(4), p. 1869-1887.

Renne P. R., Sharp W. D., Deino A. L., Orsi G., Civetta L., 1997. ${ }^{40} \mathrm{Ar} /{ }^{39} \mathrm{Ar}$ Dating into the Historical Realm: Calibration Against Pliny the Younger. Science, 277, p. 1279-1280.

Rosholt J.N., 1982. Mobilization and weathering. In $U$-series disequilibrium: applications to environmental problems, Ivanovitch and Harmon (éd.), Clarendon Press, Oxford, p. 167-180.

Rosholt J.N., Doe B.R., Tатsumoto M., 1966. Evolution of the isotopic composition of uranium and thorium in soil profiles. Geological Society of America Bulletin, 77, p. 987-1004.

Sadier B., Delannoy J.J., Benedetti L., Bourlès D.L., Jaillet S., Geneste J.M., Lebatard A.E., Arnold M., 2012. Further constraints on the Chauvet Cave artwork elaboration. PNAS, 109 (21), p. 8002-8006.

Schulman E. 1937. Some early papers on tree rings: J.C. Kapteyn. Tree-Ring Bulletin, 3(4), p. 28-29.

Shen G., Michel V., Despriée J., Han F., Granger D.E., 2012. Datation d'enfouissement par ${ }^{26} \mathrm{Al} /{ }^{10} \mathrm{Be}$ et son application préliminaire à des sites du Paléolithique Inférieur en Chine et en France $-{ }^{26} \mathrm{Al} /{ }^{10} \mathrm{Be}$ burial dating and its preliminary application to Lower Paleolithic sites in China and in France, L'Anthropologie, 116, p. 1-12.

Stallings Jr. W.S., Schulman E., Douglass A.E., 1937. Some early papers on tree-rings: I. J. Keuchler. II. J.C. Kapteyn. Tree-Ring Bulletin, 3(4), p. 27-28.

Synal H.A., Stocker M., Suter M., 2007. MICADAS: a new compact radiocarbon AMS system, Nuclear Instruments and Methods in Physics Research Section B, 259, p. 7-13.

Tessier L., 2010. Introduction scientifique, La dendrochronologie : retour vers le passé. In Laurent Astrade et Cécile Miramont (dir.), Panorama de la dendrochronologie en France, Edytem, n ${ }^{\circ} 11$, p. 13-18. 
Thellier E., 1938. Sur l'aimantation des terres cuites et ses applications géophysiques. Annales de l'Institut de Physique du Globe de Paris, 16, p. 157-302.

Thellier E., 1966. Le champ magnétique fossile. Nucleus, 7 , p. 1-35.

Thellier E., 1981. Sur la direction du champ magnétique terrestre en France durant les deux derniers millénaires. Physics of the Earth and Planetary Interiors, 24, p. 89-132.

Thellier E., Thellier O., 1959. Sur l'intensité du champ magnétique terrestre dans le passé historique et géologique. Annales de Geophysique, 15, p. 285-376.

Tissoux H., Toyoda S., Falguères C., Voinchet P., Takada M., BAHAin, J.J., Despriée J., 2008. ESR dating of quartz from two different Pleistocene deposits using $\mathrm{Al}$ and Ti-centers. Geochronometria 30, p. 23-31.

Toffolo M. B., Regev L., Mintz E., Poduska K. M., ShahackGross R., Berthold C., Miller C. E., Boaretto E., 2017. Accurate radiocarbon dating of archaeological ash using pyrogenic aragonite. Radiocarbon, 59, p. 231-249.

Toyoda S., Voinchet P., Falguères C., Dolo J.-M., Laurent M., 2000. Bleaching of ESR signals by the sunlight: a laboratory experiment for establishing the ESR dating of sediments. Applied Radiation and Isotopes, 52(5), p. 1357-1362.

Tribolo C., Mercier N., Valladas H., Joron J.-L., Guibert P., Lefrais Y., Selo M., Texier P.-J., Rigaud J.-P., Porraz G., Poggepoel C., Parkington J.-E., Texier J.-P., Lenoble A., 2009. Thermoluminescence dating of a Stillbay-Howiesons Poort sequence at Diepkloof Rock Shelter (Western Cape,
South Africa). Journal of Archaeological Sciences, 36, p. 730739.

Urbanová P., Guibert P., 2017. Methodological study on single grain OSL dating of mortars: Comparison of five reference archaeological sites. Geochronometria, 44, 77-97.

Valladas H., Mercier N., Falguères Ch., Bahain J.-J., 1999. Contribution des méthodes nucléaires à la chronologie des cultures paléolithiques entre 300000 et 35000 ans BP. Gallia Préhistoire, 41, p. 153-166.

Valladas H., Tisnérat-Laborde N., Cacher H., Kaltnecker É., Arnold M. Oberlin C., Évin J., 2005. Bilan des datations carbone 14 effectuées sur des charbons de bois de la grotte Chauvet. Bulletin de la Société Préhistorique Française, 102 (1), p. 109-113.

Veitch R.J., Hedley I.G., Wagner J.J., 1984. An investigation of the intensity of the geomagnetic field during Roman times using magnetically anisotropic bricks and tiles. Archives des Sciences de Genève, 37 (3), p. 359-373.

Voinchet P., Despriée J., Tissoux H., Falguères C., Bahain J.-J., Gageonnet R., Dépont J., Dolo J.-M., 2010. ESR chronology of alluvial deposits and first human settlements of the Middle Loire Basin (Region Centre, France). Quaternary Geochronology, 5, p. 381-384.

Xu B., Toffolo M. B., Boaretto E., Poduska K. M., 2016. Assessing local and long-range structural disorder in aggregatefree lime binders. Industrial \& Engineering Chemistry Research, 55, p. $8334-8340$. 